\title{
Essential Oil of Pinus koraiensis Exerts Antiobesic and Hypolipidemic Activity via Inhibition of Peroxisome Proliferator-Activated Receptors Gamma Signaling
}

\author{
Hyun-Suk Ko, ${ }^{1}$ Hyo-Jeong Lee, ${ }^{1}$ Hyo-Jung Lee, ${ }^{1,2}$ Eun Jung Sohn, ${ }^{1}$ Miyong Yun, \\ Min-Ho Lee, ${ }^{3}$ and Sung-Hoon Kim ${ }^{1}$ \\ ${ }^{1}$ College of Oriental Medicine, Kyung Hee University, 1 Hoegi-dong, Dongdaemun-gu, Seoul 130-701, Republic of Korea \\ ${ }^{2}$ Medical Genomics Research Center, Korea Research Institute of Bioscience and Biotechnology, Daejeon 305-811, Republic of Korea \\ ${ }^{3}$ College of Life Sciences and Biotechnology, Kyung Hee University, Seoul 130-701, Republic of Korea
}

Correspondence should be addressed to Sung-Hoon Kim; sungkim7@khu.ac.kr

Received 16 April 2013; Accepted 8 July 2013

Academic Editor: Jae Youl Cho

Copyright (C) 2013 Hyun-Suk Ko et al. This is an open access article distributed under the Creative Commons Attribution License, which permits unrestricted use, distribution, and reproduction in any medium, provided the original work is properly cited.

Our group previously reported that essential oil of Pinus koraiensis (EOPK) exerts antihyperlipidemic effects via upregulation of low-density lipoprotein receptor and inhibition of acyl-coenzyme A. In the present study, we investigated the antiobesity and hypolipidemic mechanism of EOPK using in vitro 3T3-L1 cells and in vivo HFD-fed rats. EOPK markedly suppressed fat accumulation and intracellular triglyceride associated with downregulation of adipogenic transcription factor expression, including PPAR $\gamma$ and CEBP $\alpha$ in the differentiated 3T3-L1 adipocytes. Additionally, EOPK attenuated the expression levels of FABP and GPDH as target genes of PPAR $\gamma$ during adipocyte differentiation. Furthermore, PPAR $\gamma$ inhibitor GW9662 enhanced the decreased expression of FABP and PPAR $\gamma$ and fat accumulation induced by EOPK. To confirm the in vitro activity of EOPK, animal study was performed by administering normal diet, HFD, and/or EOPK at the dose of 100 or $200 \mathrm{mg} / \mathrm{kg}$ for 6 weeks. Consistently, EOPK significantly suppressed body weight gain, serum triglyceride, total cholesterol, LDL cholesterol, and AI value and increased HDL cholesterol in a dose-dependent manner. Immunohistochemistry revealed that EOPK treatment abrogated the expression of PPAR $\gamma$ in the liver tissue sections of EOPK-treated rats. Taken together, our findings suggest that EOPK has the antiobesic and hypolipidemic potential via inhibition of PPAR $\gamma$-related signaling.

\section{Introduction}

Obesity as a medical condition of excess body fat caused by an accumulation of adipose tissue mass [1] is tightly associated with various health disorders such as hyperlipidemia, diabetes, and cardiovascular diseases [2]. In obesity, adipocytes accumulate excessive fat and adipocytokine production is disrupted [3,4]. Adipose tissue development observed in obese individuals is closely related to hypertrophy and hyperplasia, the latter involving proliferation and differentiation of preadipocytes to adipocytes. The peroxisome proliferatoractivated receptor (PPAR) and CCAAT/enhancer-binding proteins (C/EBP) families of transcription factors regulate this adipocyte differentiation [5-8]. These transcriptional factors regulate the expression of genes involved in the induction adipocyte phenotypes [5].

Pharmaceutical antiobesity drugs are generally developed to loss or control body weight. Although various prescribed or nonprescribed medications have been used for treatment of obesity patients, there is a limitation to apply long-term use because of their severe side effects. Only orlistat (Xenical) has been approved by the FDA for long-term use. Recently, many studies reported antiobesity activity of natural products and suggested the potential as antiobesity agents or their supplements.

Pinus koraiensis (Phylum Pinophyta, Class Pinopsida, Order Pinales, Family Pinaceae) is an evergreen tree found in Korea, China, far eastern Russia, and central Japan. The major 
bioactive chemicals from $P$. koraiensis include camphene, Dlimonene, $\alpha$-pinene, borneol, $\beta$-pinene, 4 -carene, bicyclohept-3-ene, 3-carene, $\beta$-phellandrene, and fencyl [9]. We and others demonstrated the biological efficacies and genotoxicity of essential oil from $P$. koraiensis seed, leaf, nut, and cone [9-13]. However, the biological and biochemical effects of essential oil from $P$. koraiensis have not been reported yet.

Recently, many studies reported that natural products such as Rhizoma Polygonati falcatum (RPF) [14], Boussingaultia gracilis Miers var. pseudobaselloides Bailey [15], Chinese black tea (Pu-erh tea) extract, and gallic acid [16] showed the potential of antiobesity activity in vitro or in vivo. In the present study, we investigated the antiobesic and hypolipidemic effect of essential oil of $P$. koraiensis SIEB (EOPK) in 3T3-L1 cells by Oil-Red $\mathrm{O}$ staining, measuring triglyceride, and analyzing expression levels of adipogenic factors and in high-fat diet-fed rats by measuring body weights and fats, and lipid metabolites.

\section{Materials and Methods}

2.1. Preparation of Essential Oil of P. koraiensis Leaves (EOPK). EOPK was prepared using the hydrodistillation method [17]. Driedand pulverized $P$. koraiensis leaves were immersed in distilled water and submitted to steam distillation using an apparatus with a condenser (Hanil Labtech, Seoul, Korea). The distillation continued for $3-4 \mathrm{~h}$ at $90^{\circ} \mathrm{C}$, and then the volatile compounds contained in the water-soluble fraction were allowed to settle for $20 \mathrm{~min}$. The essential oil layer was separated and purified through microfiltration.

2.2. Cell Culture. 3T3-L1 preadipocytes were purchased from Korean Cell Line Bank (KCLB). The cells were cultured in Dulbecco's Modified Eagle's Medium (DMEM) with 10\% FBS in a humidified atmosphere of $95 \%$ air and $5 \% \mathrm{CO}_{2}$ at $37^{\circ} \mathrm{C}$.

2.3. Cytotoxicity Assay. The cytotoxicity of EOPK against 3T3-L1 cells was measured by 3-(4,5-dimethyl-2-thiazolyl)2,5-diphenyl-2H-tetrazolium bromide (MTT) colorimetric assay. The cells were seeded onto 96-well microplates at a density of $1 \times 10^{4}$ cells per well and treated with various concentrations of EOPK $(0,12.5,25$, or $50 \mu \mathrm{g} / \mathrm{mL})$ for $24 \mathrm{~h}$. MTT working solution was then added to the microplates at $37^{\circ} \mathrm{C}$ for $2 \mathrm{~h}$, and then MTT extraction buffer (20\% SDS and $50 \%$ dimethylformamide) was added at $37^{\circ} \mathrm{C}$ overnight. Optical density (OD) was measured using microplate reader (Sunrise, TECAN, Mannedorf, Switzerland) at $570 \mathrm{~nm}$. Cell viability was calculated by employing the following equation: cell viability $(\%)=[\mathrm{OD}(\mathrm{EOPK})-\mathrm{OD}($ Blank$)] /[\mathrm{OD}(\mathrm{Con}-$ trol) - OD (Blank)] $\times 100$.

2.4. Differentiation Induction and Oil-Red O Staining. The preadipocyte 3T3-L1 cells were plated onto 6-well plates on day 0 and incubated to confluent status. For adipocyte differentiation, the confluent cells were treated with $1 \mu \mathrm{M}$ dexamethasone, $1 \mu \mathrm{g} / \mathrm{mL}$ insulin, and $0.5 \mathrm{mM}$ IBMX for 2 days, and the medium was replaced by fresh normal medium only containing $1 \mu \mathrm{g} / \mathrm{mL}$ insulin. On day 8 , the differentiated adipocyte cells were cultured in the presence or absence of EOPK $(100 \mu \mathrm{g} / \mathrm{mL})$ for 2 days. The cells were fixed with $2 \%$ paraformaldehyde, washed twice with PBS, and finally stained with Oil-Red. After dissolving, cellular-lipid retained Oil-Red $\mathrm{O}$ in isopropanol, and adipocyte expression was estimated by measuring OD using microplate reader (Sunrise, TECAN, Mannedorf, Switzerland) at $510 \mathrm{~nm}$.

2.5. RT-PCR Analysis. The 3T3-L1 cells were treated with or without EOPK (50 or $100 \mathrm{mg} / \mathrm{mL}$ ) for $24 \mathrm{~h}$. The total RNA was extracted by using TRIzol reagent (Invitrogen, Carlsbad, CA, USA) according to the manufacturer's instructions. cDNA was synthesized from $1 \mu \mathrm{g}$ of total RNA and subjected to PCR reaction by using a SuperScript One-Step reverse transcription-PCR (RT-PCR) kit (Invitrogen, Carlsbad, CA, USA). The PCR conditions were 30 cycles of $94^{\circ} \mathrm{C}$ for $30 \mathrm{~s}$, $57^{\circ} \mathrm{C}$ for $30 \mathrm{~s}$, and $72^{\circ} \mathrm{C}$ for $30 \mathrm{~s}$. The primer sequences were as follows: PPAR $\gamma$ (forward $5^{\prime}$-GGTGAAACTCTGGGAGATTC- $3^{\prime}$ and reverse $5^{\prime}$-CAACCATTGGGTCAGCTCTT- $3^{\prime}$ ); C/EBP $\alpha$ (forward $5^{\prime}$-AGGTGCTGGAGTTGACCAGT- $3^{\prime}$ and reverse $5^{\prime}$-CAGCCTAGAGATCCAGCGAC- $3^{\prime}$ ); GPDH (forward $5^{\prime}$-GAACTAAGGAGCAGCTCAAAGGTTC- $3^{\prime}$ and reverse $5^{\prime}$-CAGTTGACTGACTGAGCAAACATAG- $3^{\prime}$ ); $\beta$-actin (forward $5^{\prime}$-ACCGTGAAAAGATGACCCAG- $3^{\prime}$ and reverse $5^{\prime}$-TACGGATGACAACGTCACAC- $3^{\prime}$ ). PCR products were run on $2 \%$ agarose gel and then stained with ethidium bromide. Stained bands were visualized under UV light and photographed.

2.6. Western Blotting. Whole cell lysates $(25 \mu \mathrm{g})$ were prepared using lysis buffer (20 mM Tris, pH 7.4, $250 \mathrm{mM} \mathrm{NaCl}$, 2 mM EDTA, pH 8.0, 0.1\% Triton X-100, $0.01 \mathrm{mg} / \mathrm{mL}$ aprotinin, $0.003 \mathrm{mg} / \mathrm{mL}$ leupeptin, $0.4 \mathrm{mM}$ phenylmethylsulfonyl fluoride (PMSF), and $4 \mathrm{mM} \mathrm{NaVO} 4$ ). The lysates were spun at $13000 \times \mathrm{g}$ for $15 \mathrm{~min}$ to remove insoluble material and resolved on a $10 \%$ SDS-PAGE gel. After electrophoresis, the proteins were electrotransferred to a nitrocellulose membrane, blocked with $5 \%$ nonfat milk, and probed with antibodies against PPAR $\gamma$ (Novus, Littleton, CO, USA), C/EBP $\alpha$ (Cell Signaling Tech., Danvers, MA, USA), FABP (Cell Signaling Tech., Danvers, MA, USA), and $\beta$-actin (Sigma, St. Louis, MO, USA) overnight.

The blots were washed, exposed to horseradish peroxidase- (HRP-) conjugated secondary antibodies for $2 \mathrm{~h}$, and finally examined by enhanced chemiluminescence (ECL) (GE Health Care Bio-Sciences, Piscataway, NJ, USA).

2.7. Animals, Diet Manipulation, and EOPK Treatment. Male Sprague-Dawley rats, at 4 weeks of age, were purchased from Hyo-Chang Science (Daegu, Korea) and maintained under conventional conditions. Fifty rats were divided into four groups; normal group (low fat diet), control group (high-fat diet), two EOPK-treated groups- and atorvastatin (positive control) treated group consuming high-fat diets (10 rats per group). Rats were fed the normal (low fat) diet (group 1) or the high-fat diet (groups 2-5) for 6 weeks. High-fat diet composition was described in Table 1. For EOPK treatment, 
TABLE 1: Composition of basal and high-fat diet.

\begin{tabular}{|c|c|c|}
\hline Ingredient & Basal diet (\%) & High-fat diet (\%) \\
\hline Casein & 20.0 & 20.0 \\
\hline DL-Methionine & 0.3 & 0.3 \\
\hline Corn starch & 15.0 & 15.0 \\
\hline Sucrose & 50.0 & 34.5 \\
\hline Fiber $^{1}$ & 5.0 & 5.0 \\
\hline Corn oil & 5.0 & - \\
\hline AIN-mineral mixture ${ }^{2}$ & 3.5 & 3.5 \\
\hline AIN-vitamin mixture ${ }^{3}$ & 1.0 & 1.0 \\
\hline Choline bitartrate & 0.2 & 0.2 \\
\hline Beef tallow & - & 20.5 \\
\hline
\end{tabular}

${ }^{1}$ Cellulose: Sigma Co. Ltd., USA.

${ }^{2}$ Mineral mixture, based on Rogers and Haper [18], contained the following $(\mathrm{g} / \mathrm{kg}$ diet): calcium phosphate dibasic 200.0, sodium chloride 74.0 , potassium citrate monohydrate 220.0 , potassium sulfate 52.0 , magnesium oxide 24.0 , magnesium carbonate 3.5 , ferric citrate 6.0 , zinc carbonate 1.6 , cupuric carbonate 0.3 , potassium iodate 0.01 , chromium potassium sulfate 0.55 , sucrose, and finely powered make 1,000 .

${ }^{3}$ Vitamin mixture (g/kg diet): thiamine $\mathrm{HCl} 0.6$, biotin 0.02 , riboflavin 0.6 , cyanocobalamin 0.001 , pyridoxine $\mathrm{HCl} 0.7$, retinyl acetate 0.8 , nicotinic acid 3.0, DL-tocopherol 3.8, Ca-pantothenate 1.6, 7-dehydrocholesterol 0.0025, folic acid 0.2 , methionine 0.005 , sucrose, and finely powered make 1,000 .

EOPK dissolved in $4 \%$ tween 80 /normal saline was orally administered once daily to the rats at the doses of 100 (group 3) and $200 \mathrm{mg} / \mathrm{kg}$ (group 4) for 6 weeks from the 1st day of high-fat diet feeding, whereas PBS was orally administered to the rats in control group (group 2). As a positive control, atorvastatin was administered at a dose of $10 \mathrm{mg} / \mathrm{kg}$ (group 5).

2.8. Preparation of Rat Serum. Whole blood was collected from rats by cardiac puncture method, and serum was isolated by centrifugation at $3000 \mathrm{rpm}$ for $10 \mathrm{~min}$.

2.9. Measurement of Total Cholesterol Level. Total cholesterol level was measured by using a total cholesterol assay kit (AM 202-K, Asan Pharm Co., Seoul, Korea) based on Richmond's method [19].

2.10. Measurement of Triglyceride Level. Triglyceride level was measured by a triglyceride assay kit (AM 157S-K, Asan Pharm Co., Seoul, Korea) based on McGowan's method [20].

2.11. Measurement of Serum HDL and LDL Levels. The levels of high-density lipoprotein (HDL) and low-density lipoprotein (LDL) in serum were measured using Roche cobas C-111 analyzer (Roche-Diagnostics, Indianapolis, IN, USA): AI was calculated by employing the following equation. $\mathrm{AI}=$ (total cholesterol - HDL cholesterol)/HDL cholesterol.

2.12. Measurement of Body Weight, Retroperitoneal Fat, and Epididymal Fat. The body weight of rats in normal (group 1), control (group 2), EOPK- (100 and $200 \mathrm{mg} / \mathrm{kg}$ ) treated groups (groups 3 and 4, resp.), and atorvastatin (group 5) was monitored once every week for 6 weeks. The retroperitoneal and epididymal fats were also removed and weighed from rats treated by EOPK (100 and $200 \mathrm{mg} / \mathrm{kg}$ ) or atorvastatin $(10 \mathrm{mg} / \mathrm{kg})$ on the last day of animal study.

2.13. Immunohistochemical Staining. For histopathological examination, paraffin sections $(4 \mu \mathrm{m})$ from tumors dissected were stained with hematoxylin and eosin. Immunohistochemical staining PPAR $\gamma$ (Novus, Littleton, CO, USA) was performed using the indirect avidin/biotin-enhanced horseradish peroxidase method. Antigen retrieval was performed after dewaxing and dehydration of the tissue sections by microwave for $10 \mathrm{~min}$ in $10 \mathrm{mM}$ citrate buffer. Sections were cooled to room temperature, treated with $3 \%$ hydrogen peroxide in methanol for $10 \mathrm{~min}$, and blocked with $6 \%$ horse serum for $30 \mathrm{~min}$ at room temperature in humidity chamber. Sections were then incubated with the primary antibody against PPAR $\gamma$ (diluted 1:200, Novus, Littleton, CO, USA) at $4^{\circ} \mathrm{C}$ overnight in humidity chamber. Sections were washed in PBS and incubated with secondary antibody (biotinylated goat anti-rabbit $(1: 150$, Vector laboratories, Burlingame, CA, USA) for $30 \mathrm{~min}$ in humidity chamber. After further washes, the antibodies were detected with the Vector ABC complex/horseradish peroxidase (HRP) kit (Vector Laboratories, Burlingame, CA, USA) and colordeveloped with $3,3^{\prime}$-diaminobenzidine tetrahydrochloride. For semiquantitation, ten photomicrographs $(200 \mathrm{x})$ were taken with a CCD camera, avoiding gross necrotic areas.

2.14. Statistical Analyses. All data were expressed as means \pm $\mathrm{SD}$. or SE. In vitro experiment data were analyzed by Student's $t$-test. In vivo experiment data were calculated by the analysis of variance (ANOVA) followed by Duncan's multiple range test. $P$ value of less than 0.05 was considered statistically significant. Means in the same column with different superscript letters (a, b, c, d, e, and $\mathrm{f}$ ) are significantly different $(P<0.05)$ between groups.

\section{Results}

3.1. Effect of EOPK on Fat Accumulation in 3T3-L1 AdipocyteLike Cells. Cytotoxicity of EOPK was determined by MTT assay in 3T3-L1 preadipocytes. Cells were treated with various concentrations of EOPK $(0,12.5,25$, or $50 \mu \mathrm{g} / \mathrm{mL})$ for $24 \mathrm{~h}$. As shown in Figure 1(a), EOPK had no significant effect on the viability of 3T3-L1 cells. To investigate whether EOPK can affect the cellular differentiation into adipocytes, Oil-Red $\mathrm{O}$ staining was performed in 3T3-L1 cells treated with EOPK for 8 days. The differentiated 3T3-L1 adipocytes significantly increased fat accumulation and intracellular triglyceride (Figures 1(b), 1(c), and 1(d)), when compared to preadipocytes. The fat deposits were decreased by 57 and $78 \%$ at the treatment with various concentrations of EOPK (25 or $50 \mu \mathrm{g} / \mathrm{mL}$ ), respectively, compared to untreated control, adipocytes. Results indicate that $50 \mu \mathrm{g} / \mathrm{mL}$ of EOPK was the most effective ability to inhibit adipocyte differentiation (Figures 1(b) and 1(c)). EOPK treatment reduced the level of triglyceride in cell in a dose-dependent manner (37 and 60\% 


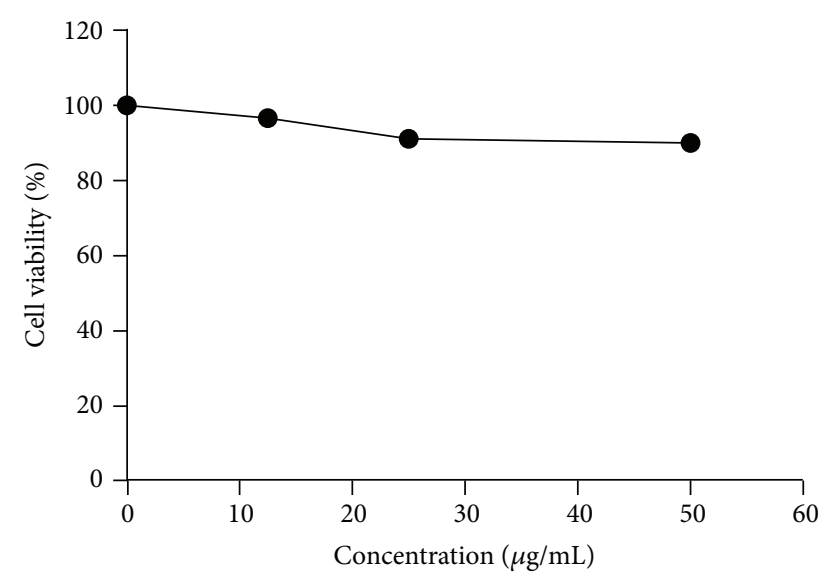

(a)
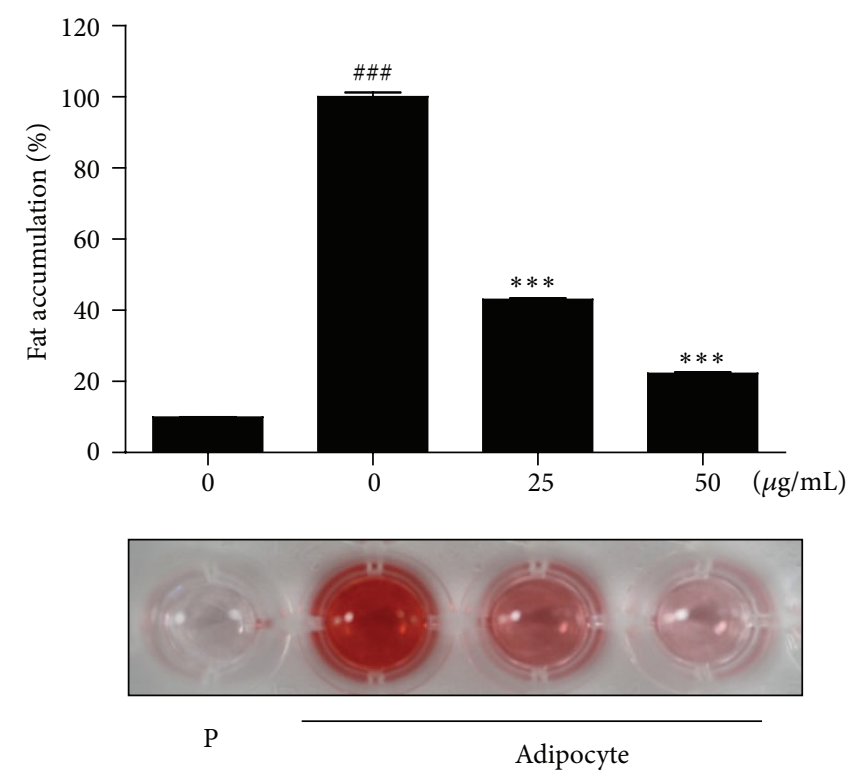

(c)

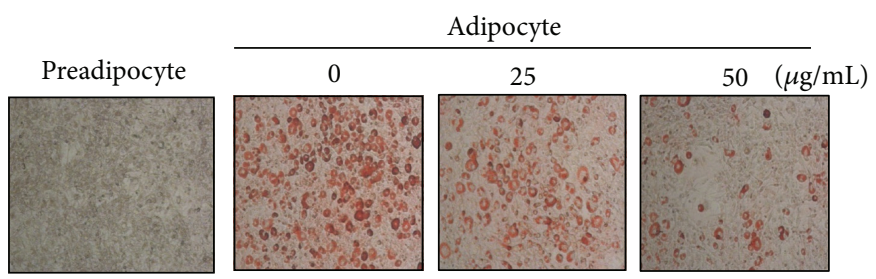

(b)

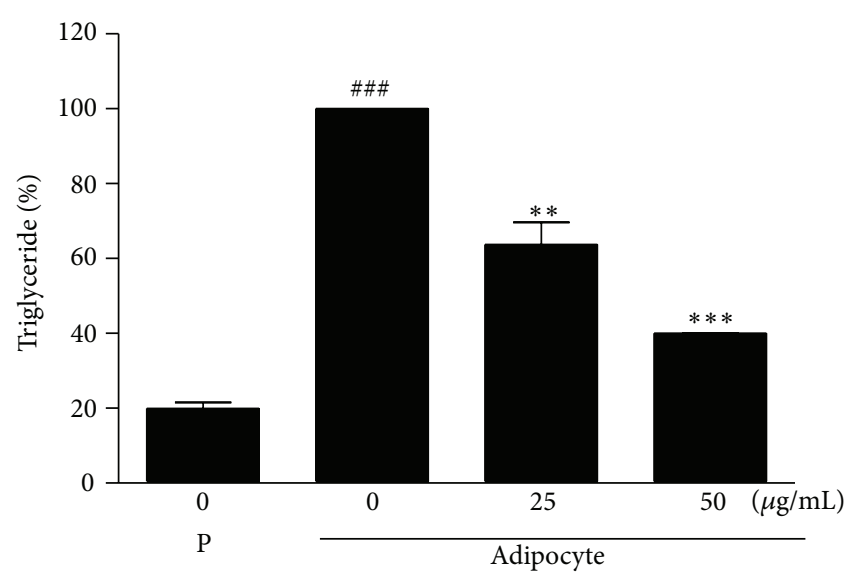

(d)

FIGURE 1: Effect of EOPK on the differentiation of 3T3-L1 adipocytes. (a) Cytotoxicity of EOPK against 3T3-L1 cells was determined by MTT assay. Cells were treated with various concentrations of $\operatorname{EOPK}(0,12.5,25$, or $50 \mu \mathrm{g} / \mathrm{mL})$ for $24 \mathrm{~h}$. (b, c, and d). Confluent cells were treated with $1 \mu \mathrm{M}$ dexamethasone, $1 \mu \mathrm{g} / \mathrm{mL}$ insulin, and $0.5 \mathrm{mM}$ IBMX for 2 days, and then the medium was replaced by fresh normal medium only containing $1 \mu \mathrm{g} / \mathrm{mL}$ insulin. On day 8 , the differentiated adipocyte cells were exposed to EOPK for 2 days. (b) The differentiated cells were stained with Oil-Red $O$ dye and visualized under inverted microscopy at $\times 100$ magnifications. (c) After dissolving and cellular lipid retained Oil-Red O in isopropanol, adipocyte expression was estimated by measuring OD using microplate reader (Sunrise, TECAN, Mannedorf, Switzerland) at $510 \mathrm{~nm}$. (d) Level of intracellular triglyceride.

at concentrations of 25 and $50 \mu \mathrm{g} / \mathrm{mL}$, resp.), compared to preadipocytes (80.04\%) (Figure 1(d)).

3.2. Effect of EOPK on the Expression of C/EBP, PPAR $\gamma, G P D H$, and FABP during the Differentiation of Adipocytes. PPAR $\gamma$, $\mathrm{C} / \mathrm{EBP} \alpha, \mathrm{GPDH}$, and FABP are key factors involved in adipogenesis. In particular, $\operatorname{PPAR} \gamma$ controls adipogenic factors as a key transcription factor during adipocyte differentiation. Both mRNA and protein levels of PPAR $\gamma, \mathrm{C} / \mathrm{EBP} \alpha$, and GPDH were downregulated by EOPK in a dose-dependent manner (Figures 2(a) and 2(b)). To elucidate the underlying mechanism of EOPK, PPAR $\gamma$ antagonist GW9662 was used in 3T3-L1 adipocytes. PPAR $\gamma$ inhibitor GW9662 enhanced the decreased expression of FABP and PPAR $\gamma$ by EOPK (Figure 2(c)).

As shown in Figures 2(d) and 2(e), the lipid accumulation in 3T3-L1 adipocytes treated with EOPK and GW9662 was significantly reduced compared to untreated control. Consistently, immunohistochemistry revealed that EOPK treatment attenuated the expression of PPAR $\gamma$ in the liver tissue sections of EOPK-treated group (100 and $200 \mathrm{mg} / \mathrm{kg}$ ) compared to untreated control group (Figure 3(c)). Taken together, these results indicate that EOPK inhibits adipocyte differentiation via suppressing PPAR $\gamma$ activation. 


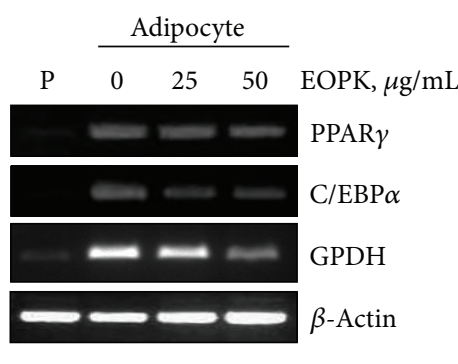

P: preadipocyte

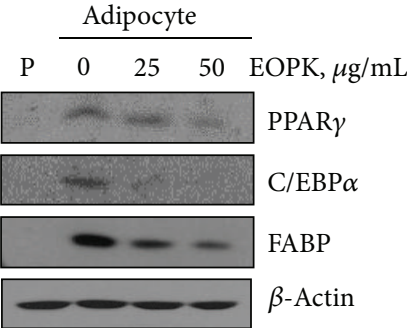

(b)

(a)

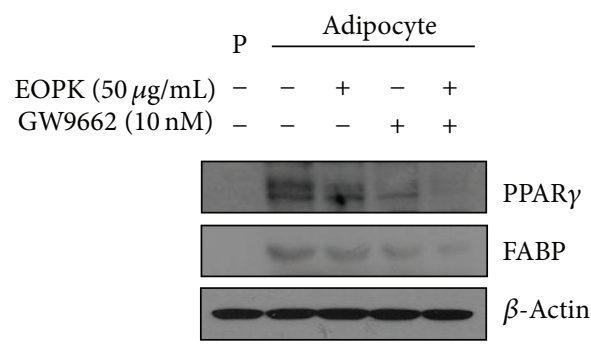

(c)

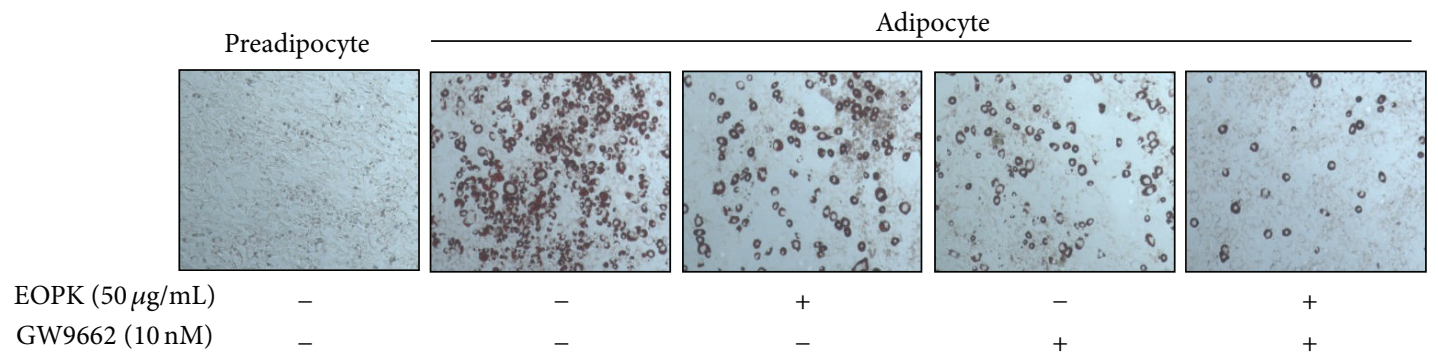

(d)

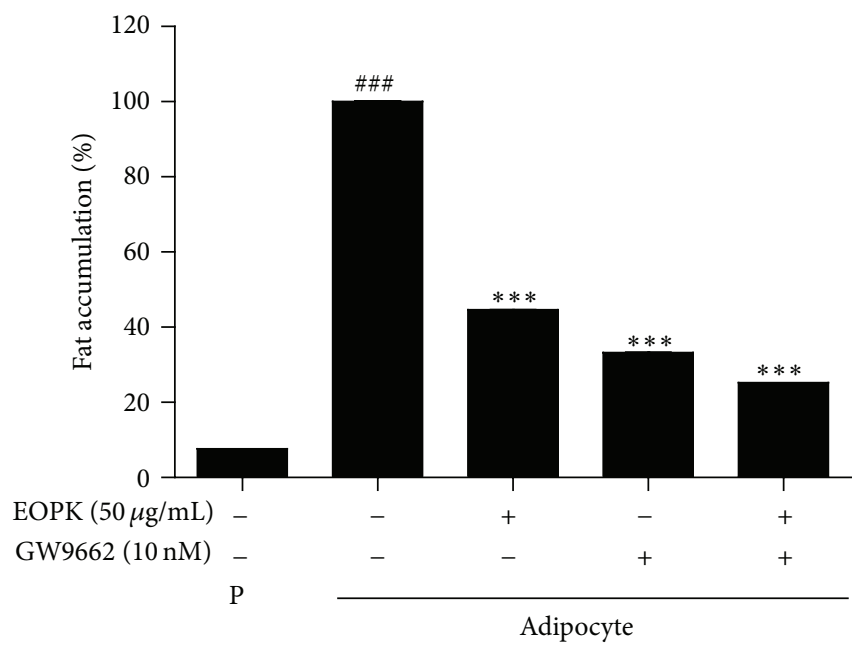

(e)

FIGURE 2: Effects of EOPK on adipocyte differentiation of 3T3-L1 preadipocytes. 3T3-L1 preadipocytes were incubated in medium containing insulin $(1.0 \mu \mathrm{g} / \mathrm{mL})$ with or without the indicated concentrations of EOPK or GW9662. (a) Total RNA was extracted from 3T3-L1 (preadipocytes or adipocytes) cells treated with EOPK and used for RT-PCR analysis of PPAR $\gamma, \mathrm{C} / \mathrm{EBP} \alpha$, GPDH, and $\beta$-actin. (b) Total proteins prepared from EOPK-treated 3T3-L1 (preadipocytes or adipocytes) cells were subjected to western blot analysis of PPAR $\gamma, \mathrm{C} / \mathrm{EBP} \alpha$ FABP, and $\beta$-actin. (c) Total proteins prepared from EOPK- or GW9662-treated 3T3-L1 (preadipocytes or adipocytes) cells were subjected to western blot analysis of PPAR $\gamma$, FABP, and $\beta$-actin. (d) Cells were fixed and stained with Oil-Red O. The Oil-Red O-stained adipocytes were photographed at a $\times 100$ magnification under a microscope. (e) Lipids were extracted using isopropanol, and the Oil-Red $\mathrm{O}$ was then analyzed at a wavelength of $520 \mathrm{~nm}$. Values are presented as means $\pm \mathrm{SE} .{ }^{*} P<0.05$ versus the control. 


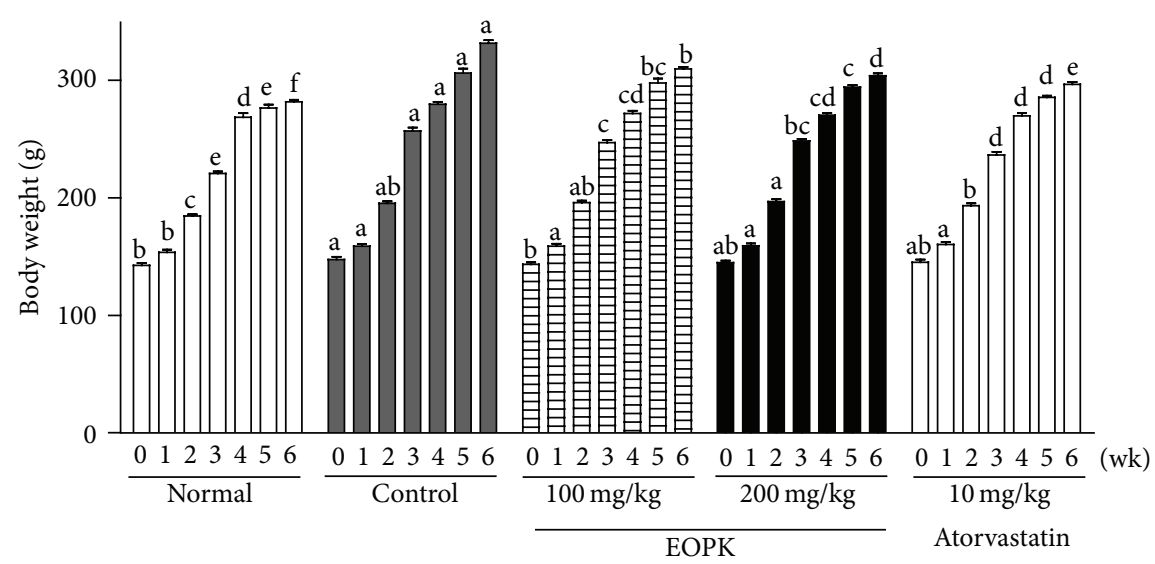

(a)

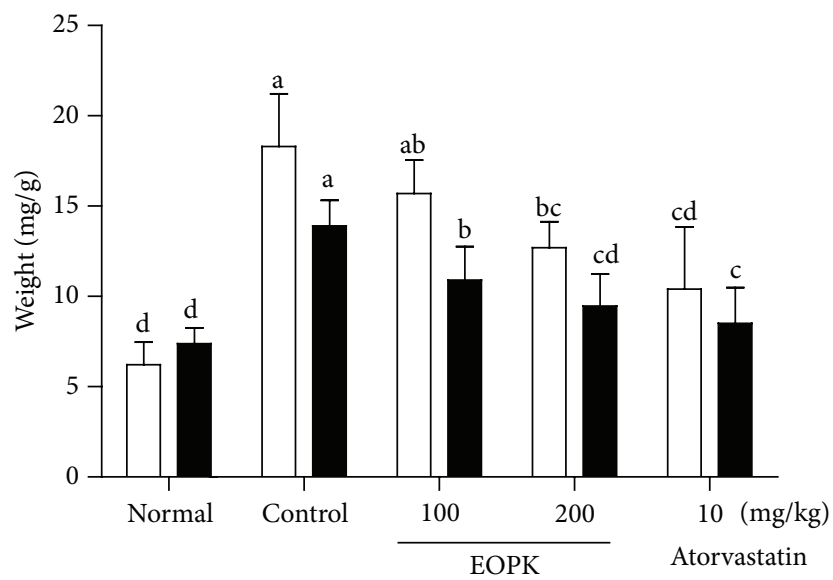

(b)

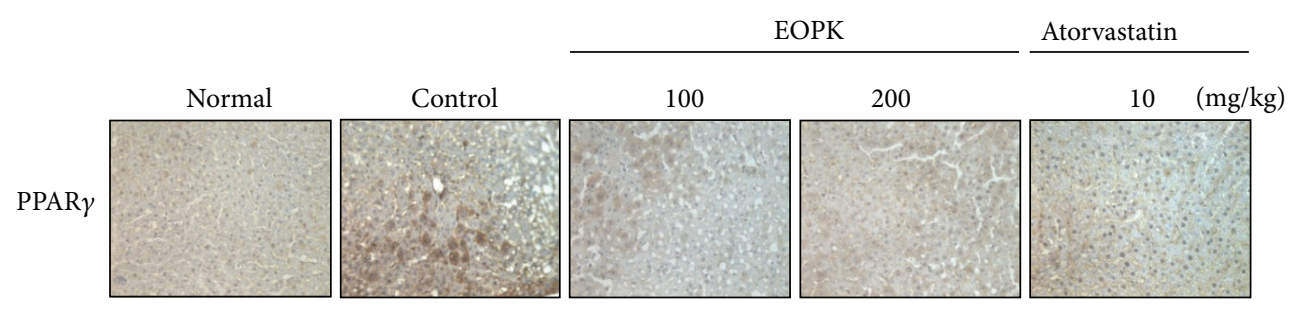

(c)

FIGURE 3: Effect of EOPK on body and abdominal fat weight of high-fat diet-fed rats. Rats fed a high diet were orally treated with or without EOPK daily for 6 consecutive weeks. (a) Body weights of rats. (b) abdominal fat weights and The retroperitoneal and epididymal fats from rats treated with or without EOPK (100 and $200 \mathrm{mg} / \mathrm{kg}$ ) were removed and weighed. (c) Representative picture of immunohistochemical staining for PPAR $\gamma$ in liver tissue sections. Data were expressed as means \pm SD. Values with the different superscript letters indicate statistical significance $(P<0.05)$ between groups by Duncan's multiple range test.

3.3. Effect of EOPK on Body Weight of High-Fat Diet-Fed Rats. The body weights of normal (group 1), control (group 2), EOPK-treated groups (groups 3 and 4), and atorvastatintreated group (group 5) were monitored once every week for 6 weeks. As shown in Figure 3(a), the body weight of high-fat fed control group was significantly increased 2 weeks after feeding compared to normal low fat group. In contrast, the body weight gain was dose dependently abrogated in EOPK-treated groups, from the date of 3-week treatment of EOPK. Also, six weeks after treatment, the body weight was significantly gained to $331.4 \pm 2.8 \mathrm{~g}$ in high-fat fed control group compared to normal group $(281.6 \pm 1.7 \mathrm{~g})$. However, EOPK significantly suppressed the body weights to $309.5 \pm$ $1.8 \mathrm{~g}$ and $303.7 \pm 2.0 \mathrm{~g}$, respectively, at doses of $100 \mathrm{mg} / \mathrm{kg}$ and 


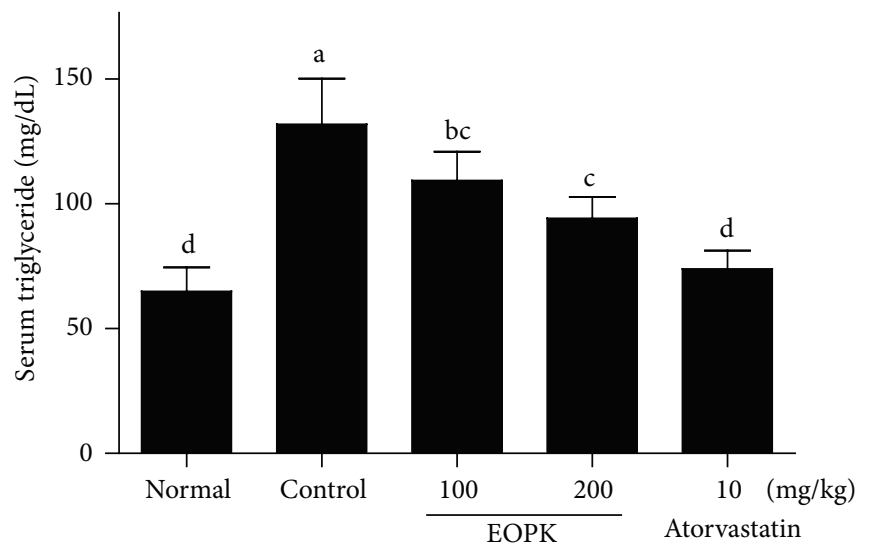

(a)

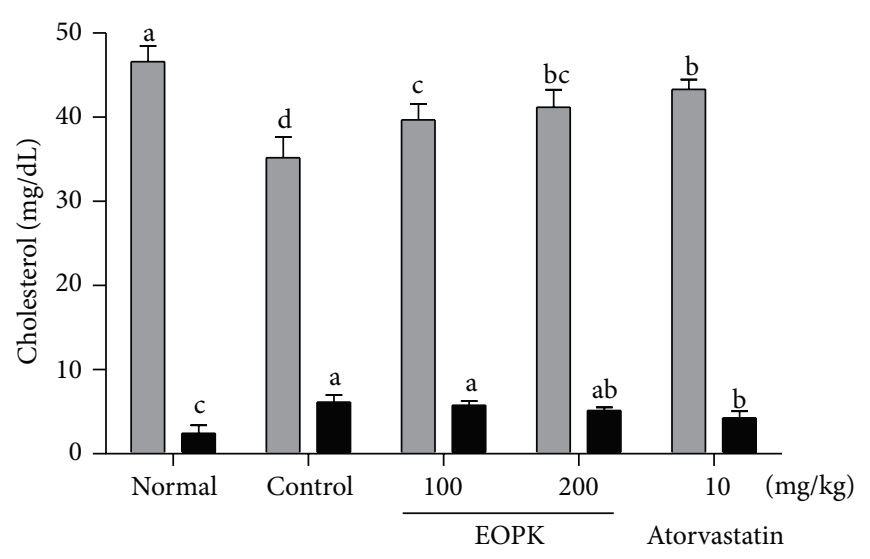

(c)

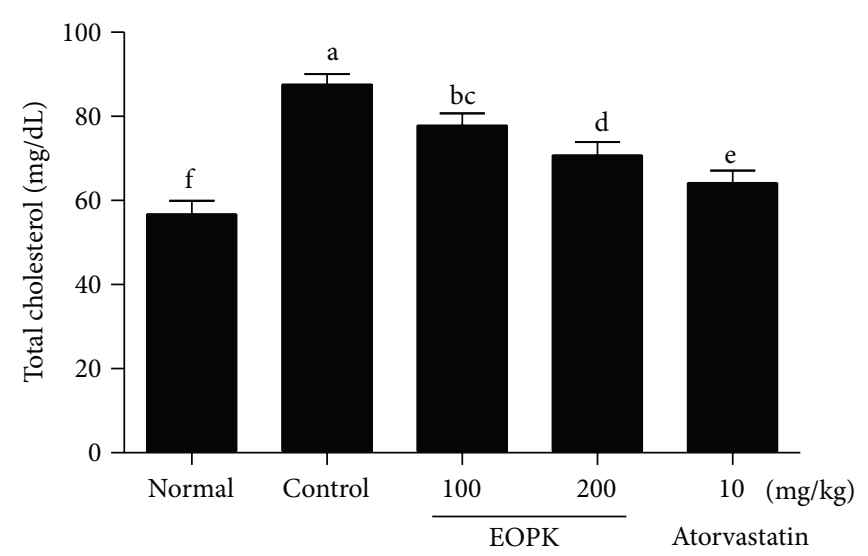

(b)

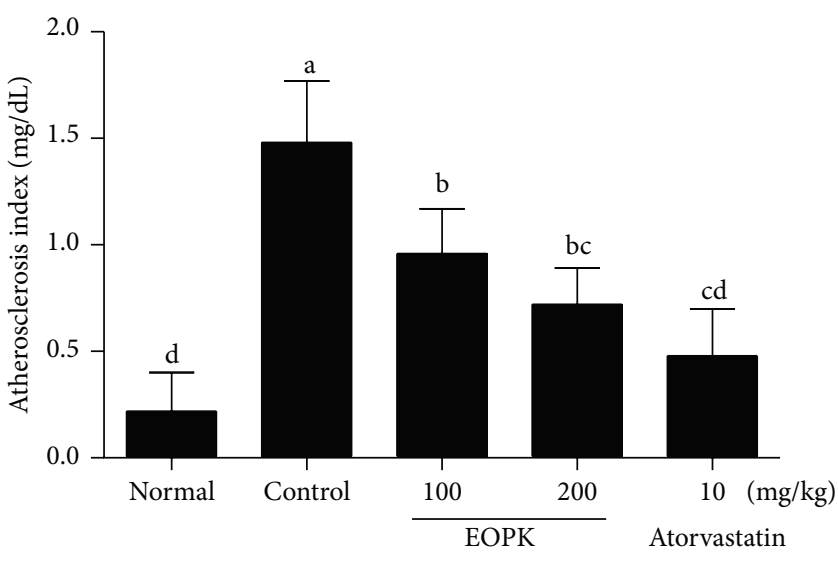

(d)

FIGURE 4: Effects of EOPK on serum lipid and cholesterol levels in high-fat diet-fed rats. (a) Triglyceride level was measured by a triglyceride assay kit (AM 157S-K, Asan Pharm Co., Seoul, Korea). (b) Total cholesterol level was measured by using a total cholesterol assay kit (AM 202-K, Asan Pharm Co., Seoul, Korea). (c) The levels of high-density lipoprotein (HDL) and low-density lipoprotein (LDL) in serum were measured using cobas c 111 analyzer (Roche-Diagnostics, Indianapolis, IN, USA). (d) AI was calculated by employing the following equation: AI = (total cholesterol - HDL cholesterol)/HDL cholesterol. Values with the different superscript letters indicate statistical significance $(P<0.05)$ between groups by Duncan's multiple range test.

$200 \mathrm{mg} / \mathrm{kg}$, almost coming up with atorvastatin as positive control $(296.5 \pm 1.9 \mathrm{~g})$.

\subsection{Effects of EOPK on Abdominal Fat Weight of High-} Fat Diet-Fed Rats. The retroperitoneal and epididymal fat weight was measured to test whether the body weight normalization by EOPK in high-fat diet-fed rats is associated with a reduction of fat content in body. In the present study, as expected, high-fat diet significantly increased the retroperitoneal fat weight to $18.3 \pm 2.91 \mathrm{mg} / \mathrm{g}$ from $6.21 \pm$ $1.26 \mathrm{mg} / \mathrm{kg}$ body weight. In contrast, EOPK decreased the retroperitoneal fat weight to $15.7 \pm 1.86$ and $12.7 \pm 1.43 \mathrm{mg} / \mathrm{g}$ at doses of 100 and $200 \mathrm{mg} / \mathrm{kg}$, respectively, compared to control group (Figure 3(b)). Likewise, epididymal fat weight was also increased in the high-fat diet-fed rats compared to normal group. Oral administration of EOPK decreased epididymal fat pad weight by $10.9 \pm 1.85$ and $8.52 \pm 1.96 \mathrm{mg} / \mathrm{g}$ at doses of 100 and $200 \mathrm{mg} / \mathrm{kg}$, respectively (Figure 3(b)).

3.5. Effects of EOPK on Serum Lipid and Cholesterol Levels in High-Fat Diet-Fed Rats. The consumption of the high-fat diet significantly increased triglyceride in control group compared to the normal low fat group (Figure 4(a)). EOPK treatment decreased level of serum triglyceride from $131.8 \pm 18.2$ to $109.3 \pm 11.5$ and $94.2 \pm 8.43 \mathrm{mg} / \mathrm{dL}$ at 100 and $200 \mathrm{mg} / \mathrm{kg}$, respectively (Figure 4(a)). Additionally, the consumption of the high-fat diet significantly increased serum total cholesterol compared to the normal low fat group, while EOPK significantly reduced the level of total cholesterol in a dosedependent manner (Figure 4(b)). The intake of the highfat diet significantly decreased level of HDL but increased level of LDL compared to normal group (Figure 4(c) control). 


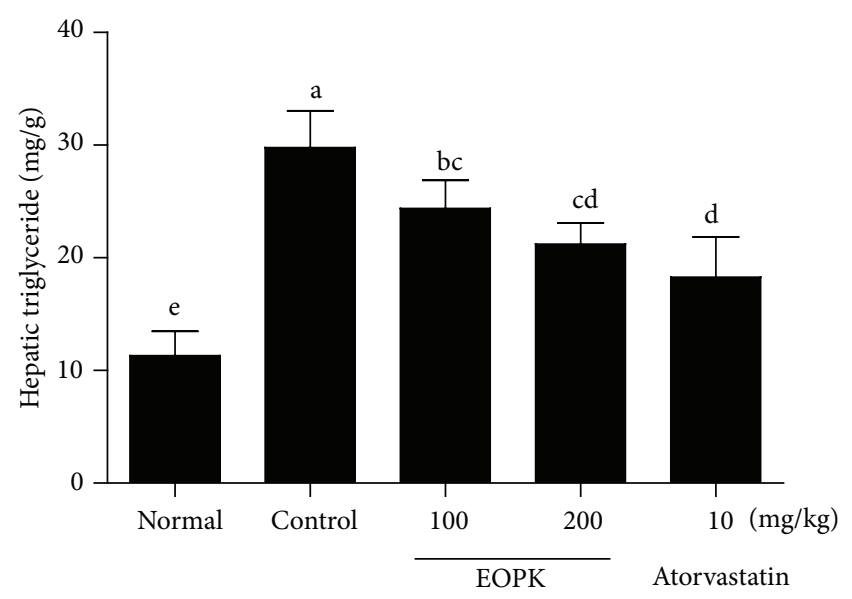

(a)

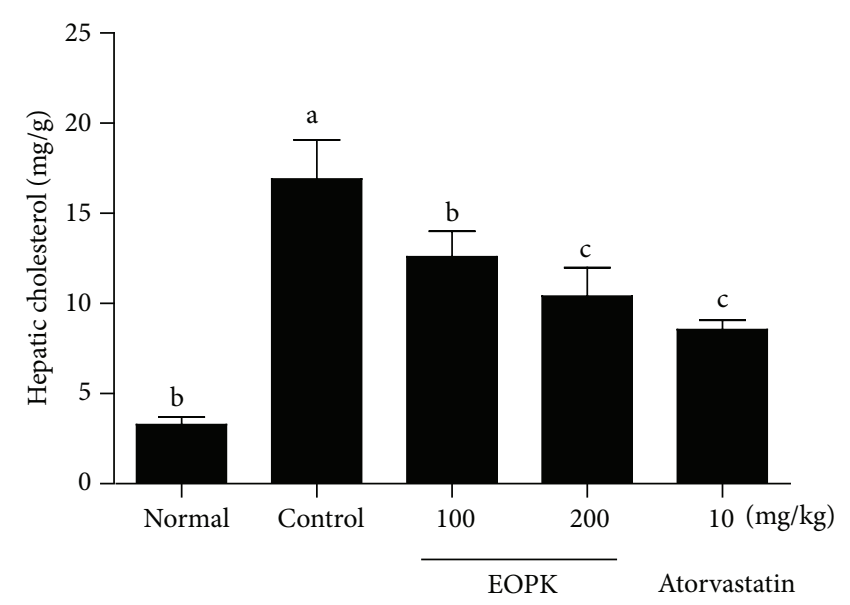

(b)

FIGURE 5: Effects of EOPK on hepatic triglyceride and cholesterol in high-fat diet-fed rats. The levels of triglyceride (a) and total cholesterol (b) in liver were measured by Biochemistry Analyzer. Values with the different superscript letters indicate statistical significance $(P<0.05)$ between groups by Duncan's multiple range test.

However, EOPK did not have a significant effect on LDL while elevating HDL level in a dose-dependent manner compared to the high-fat diet control group (Figure 4(c)). Consistently, EOPK treatment significantly decreased the atherosclerosis index (AI) value in a dose-dependent manner compared to the high-fat diet control (Figure 4(d)).

3.6. Effects of EOPK on Hepatic Triglyceride and Cholesterol in High-Fat Diet-Fed Rats. The consumption of the high-fat diet significantly increased hepatic triglyceride (Figure 5(a)) and total cholesterol (Figure 5(b)), when compared to the normal low fat group. Oral treatment with EOPK reduced the level of triglyceride from the liver in a dose-dependent manner $(24.4 \pm 2.47$ and $21.2 \pm 1.88 \mathrm{mg} / \mathrm{g}$ at doses of 100 and $200 \mathrm{mg} / \mathrm{kg}$, resp.), compared to control group (29.8 \pm $3.25 \mathrm{mg} / \mathrm{g}$ tissue) (Figure 5(a)). In addition, EOPK administration significantly lowered total cholesterol level in liver from $16.9 \pm 2.18 \mathrm{mg} / \mathrm{g}$ in the high-fat diet control group to $12.6 \pm 1.42$ and $10.4 \pm 1.59 \mathrm{mg} / \mathrm{g}$ at 100 and $200 \mathrm{mg} / \mathrm{kg}$, respectively (Figure 5(b)).

\section{Discussion}

The Korean pine ( $P$. koraiensis) is an important afforestation trees in Korea and also distributed in China, Russia, Japan, and Europe [21]. P. koraiensis extract attenuated the increase in blood pressure in spontaneously hypertensive rats [13], and its constituent pinolenic acid had cholesterollowering effect via regulation of LDL receptor activity in HepG2 hepatoma cells [22]. In addition, P. koraiensis nut oil effectively regulated satiety hormones and prospective food intake in postmenopausal overweight women [11]. Our group also reported that essential oil from $P$. koraiensis leaves had antihyperlipidemic effects via upregulation of LDL receptor and inhibition of acyl-coenzyme A: cholesterol acyltransferase [9]. In the current study, we demonstrate that EOPK has antiobesity effects in 3T3-L1 adipocytes and high-fat dietfed rat models. Adipogenesis is the process of differentiation by which undifferentiated preadipocytes are converted into differentiated adipocytes [23] and subsequently mediate the synthesis and accumulation of lipid [24]. 3T3-L1 cells are the best characterized model to study adipogenesis in vitro [25]. Oil-Red O staining revealed that EOPK significantly suppressed fat accumulation and intracellular triglyceride and decreased expression of $\operatorname{PPAR} \gamma, \operatorname{CEBP} \alpha, \mathrm{FABP}$, and GPDH in the differentiated 3T3-L1 adipocytes with no cytotoxicity, indicating the inhibitory effect of EOPK on adipocyte differentiation. Similarly, many natural compounds, including EGCG, berberine, and curcumin, suppressed PPAR $\gamma$ signaling, since PPAR $\gamma$ antagonists have been reported to effectively inhibit adipogenesis and improve insulin sensitivity in vitro and in vivo [26].

Furthermore, here the antiobesic effect of $P$. koraiensis was confirmed in high-fat diet- (HFD-) treated SD rats at the doses of 100 or $200 \mathrm{mg} / \mathrm{kg}$ via blockage of body weight gain. Since loss of body weight is mainly associated with the decrease of fat pad mass as a result of reduction of adipocyte size or triglyceride accumulation [27], our data that EOPK significantly reduced the retroperitoneal and epididymal fat weight and also serum triglyceride compared to HFD fed rats suggest that EOPK can block obesity via inhibition of lipid metabolism including triglyceride.

Cholesterol is also an important component of fat complex. In particular, low level of serum HDL cholesterol is tightly linked to the occurrence of obesity [28]. In our study, administration of EOPK significantly abrogated the contents of total cholesterol in serum. In addition, EOPK significantly increased level of HDL cholesterol in a dose-dependent manner compared to HFD control group but not LDL cholesterol. Lipid parameters in the blood can be affected 
by the hepatic metabolisms. As expected, we observed that the hepatic contents of triglyceride and cholesterol were significantly escalated in HFD fed rats compared to normal control. In contrast, EOPK treatment significantly lowered the contents of triglyceride and total cholesterol in the liver, implying that EOPK regulates lipid metabolism including triglyceride and cholesterol against obesity.

In summary, EOPK inhibited fat accumulation, intracellular triglyceride, and expression of $\operatorname{PPAR} \gamma, \mathrm{CEBP} \alpha, \mathrm{FABP}$, and GPDH in the differentiated 3T3-L1 adipocytes. Also, EOPK attenuated serum and hepatic contents of triglyceride and total cholesterol in HFD fed rat models. Furthermore, EOPK decreased the expression of PPAR $\gamma$ in the liver tissue sections of EOPK-treated rats. Overall, our findings suggest the potential of EOPK as an antiobesity agent.

\section{Conflict of Interests}

No potential conflict of interests was disclosed.

\section{Acknowledgments}

This work was supported by a grant from the NextGeneration BioGreen 21 Program (no. PJ009055) and MRC (no. 2012-0005755), Republic of Korea.

\section{References}

[1] D. W. Haslam and W. P. T. James, “Obesity," The Lancet, vol. 366, no. 9492, pp. 1197-1209, 2005.

[2] D. P. Schuster, "Obesity and the development of type 2 diabetes: the effects of fatty tissue inflammation," Diabetes, Metabolic Syndrome and Obesity, vol. 3, pp. 253-262, 2010.

[3] J. M. Friedman, "Obesity in the new millennium," Nature, vol. 404, no. 6778, pp. 632-634, 2000.

[4] J. S. Flier, "Obesity wars: molecular progress confronts an expanding epidemic," Cell, vol. 116, no. 2, pp. 337-350, 2004.

[5] F. M. Gregoire, C. M. Smas, and H. S. Sul, "Understanding adipocyte differentiation," Physiological Reviews, vol. 78, no. 3, pp. 783-809, 1998.

[6] W.-C. Yeh, Z. Cao, M. Classon, and S. L. McKnight, "Cascade regulation of terminal adipocyte differentiation by three members of the C/EBP family of leucine zipper proteins," Genes and Development, vol. 9, no. 2, pp. 168-181, 1995.

[7] G. J. Darlington, S. E. Ross, and O. A. MacDougald, "The role of C/EBP genes in adipocyte differentiation," Journal of Biological Chemistry, vol. 273, no. 46, pp. 30057-30060, 1998.

[8] M. I. Lefterova and M. A. Lazar, "New developments in adipogenesis," Trends in Endocrinology and Metabolism, vol. 20, no. 3, pp. 107-114, 2009.

[9] J.-H. Kim, H.-J. Lee, S.-J. Jeong, M.-H. Lee, and S.-H. Kim, "Essential oil of pinus koraiensis leaves exerts antihyperlipidemic effects via up-regulation of low-density lipoprotein receptor and inhibition of acyl-coenzyme a: cholesterol acyltransferase," Phytotherapy Research, vol. 26, no. 9, pp. 1314-1319, 2012.

[10] X. Chen, Y. Zhang, Z. Wang, and Y. Zu, "Invivo antioxidant activity of Pinus koraiensis nut oil obtained by optimised supercritical carbon dioxide extraction," Natural Product Research, vol. 25, no. 19, pp. 1807-1816, 2011.
[11] W. J. Pasman, J. Heimerikx, C. M. Rubingh et al., "The effect of Korean pine nut oil on in vitro CCK release, on appetite sensations and on gut hormones in post-menopausal overweight women," Lipids in Health and Disease, vol. 7, article 10, 2008.

[12] G. J. A. Speijers, L. H. T. Dederen, and H. Keizer, "A sub-chronic (13 weeks) oral toxicity study in rats and an in vitro genotoxicity study with Korean pine nut oil (PinnoThin TG)," Regulatory Toxicology and Pharmacology, vol. 55, no. 2, pp. 158-165, 2009.

[13] M. Sugano, I. Ikeda, K. Wakamatsu, and T. Oka, "Influence of Korean pine (Pinus koraiensis)-seed oil containing cis-5,cis9,cis-12-octadecatrienoic acid on polyunsaturated fatty acid metabolism, eicosanoid production and blood pressure of rats," British Journal of Nutrition, vol. 72, no. 5, pp. 775-783, 1994.

[14] U. H. Park, J. C. Jeong, J. S. Jang et al., "Negative regulation of adipogenesis by kaempferol, a component of Rhizoma Polygonati falcatum in 3T3-L1 cells," Biological \& Pharmaceutical Bulletin, vol. 35, no. 9, pp. 1525-1533, 2012.

[15] H. Kim and S. Y. Choung, "Anti-obesity effects of Boussingaulti gracilis Miers var. pseudobaselloides Bailey via activation of AMP-activated protein kinase in 3T3-L1 cells," Journal of Medicinal Food, vol. 15, no. 9, pp. 811-817, 2012.

[16] Y. Oi, I.-C. Hou, H. Fujita, and K. Yazawa, "Antiobesity effects of Chinese black tea (Pu-erh tea) extract and gallic acid," Phytotherapy Research, vol. 26, no. 4, pp. 475-481, 2012.

[17] E.-J. Hong, K.-J. Na, I.-G. Choi, K.-C. Choi, and E.-B. Jeung, "Antibacterial and antifungal effects of essential oils from coniferous trees," Biological and Pharmaceutical Bulletin, vol. 27, no. 6, pp. 863-866, 2004.

[18] Q. R. Rogers and A. E. Harper, "Amino acid diets and maximal growth in the rat," Journal of Nutrition, vol. 87, no. 3, pp. 267273, 1965.

[19] W. Richmond, "Use of cholesterol oxidase for assay of total and free cholesterol in serum by continuous flow analysis," Clinical Chemistry, vol. 22, no. 10, pp. 1579-1588, 1976.

[20] M. W. McGowan, J. D. Artiss, D. R. Strandbergh, and B. Zak, "A peroxidase-coupled method for the colorimetric determination of serum triglycerides," Clinical Chemistry, vol. 29, no. 3, pp. 538-542, 1983.

[21] D. S. Choi, M. Kayama, H. O. Jin, C. H. Lee, T. Izuta, and T. Koike, "Growth and photosynthetic responses of two pine species (Pinus koraiensis and Pinus rigida) in a polluted industrial region in Korea," Environmental Pollution, vol. 139, no. 3, pp. 421-432, 2006.

[22] J.-W. Lee, K.-W. Lee, S.-W. Lee, I.-H. Kim, and C. Rhee, "Selective increase in pinolenic acid (all-cis-5,9,12-18:3) in Korean pine nut oil by crystallization and its effect on LDL-receptor activity," Lipids, vol. 39, no. 4, pp. 383-387, 2004.

[23] T. C. Otto and M. D. Lane, "Adipose development: from stem cell to adipocyte," Critical Reviews in Biochemistry and Molecular Biology, vol. 40, no. 4, pp. 229-242, 2005.

[24] A. G. Cristancho and M. A. Lazar, "Forming functional fat: a growing understanding of adipocyte differentiation," Nature Reviews Molecular Cell Biology, vol. 12, no. 11, pp. 722-734, 2011.

[25] H. Green and O. Kehinde, "An established preadipose cell line and its differentiation in culture. II. Factors affecting the adipose conversion," Cell, vol. 5, no. 1, pp. 19-27, 1975.

[26] J. Rieusset, F. Touri, L. Michalik et al., "A new selective peroxisome proliferator-activated receptor $\gamma$ antagonist with antiobesity and antidiabetic activity," Molecular Endocrinology, vol. 16, no. 11, pp. 2628-2644, 2002.

[27] M. Rosenbaum, R. L. Leibel, and J. Hirsch, "Obesity," The New England Journal of Medicine, vol. 337, no. 6, pp. 396-407, 1997. 
[28] S. C. Woods, R. J. Seeley, D. Porte Jr., and M. W. Schwartz, "Signals that regulate food intake and energy homeostasis," Science, vol. 280, no. 5368, pp. 1378-1383, 1998. 


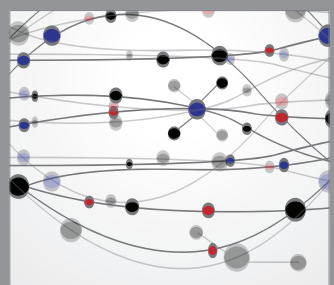

The Scientific World Journal
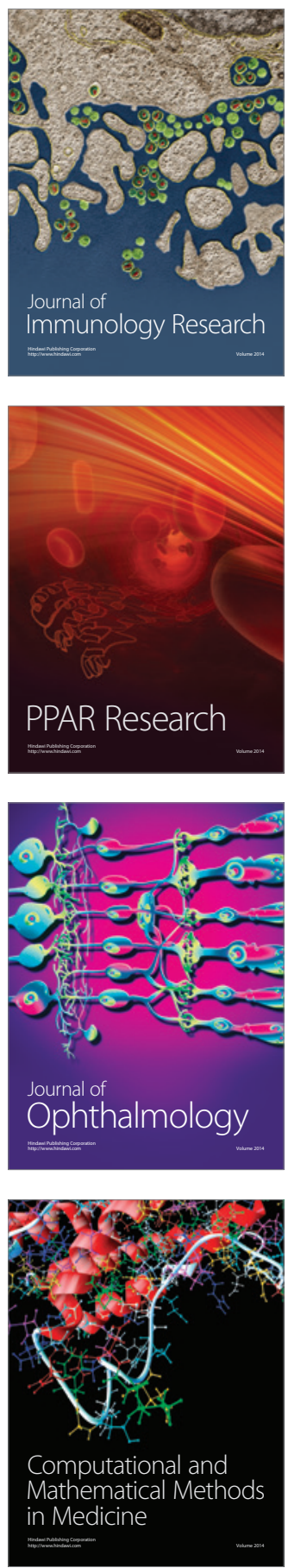

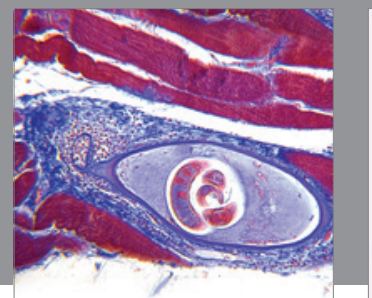

Gastroenterology

Research and Practice
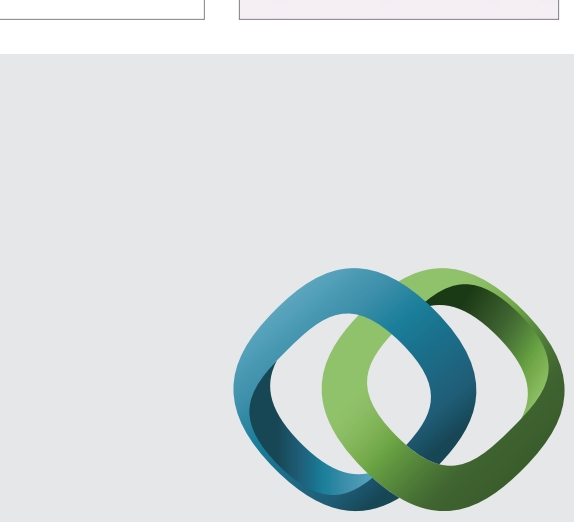

\section{Hindawi}

Submit your manuscripts at

http://www.hindawi.com
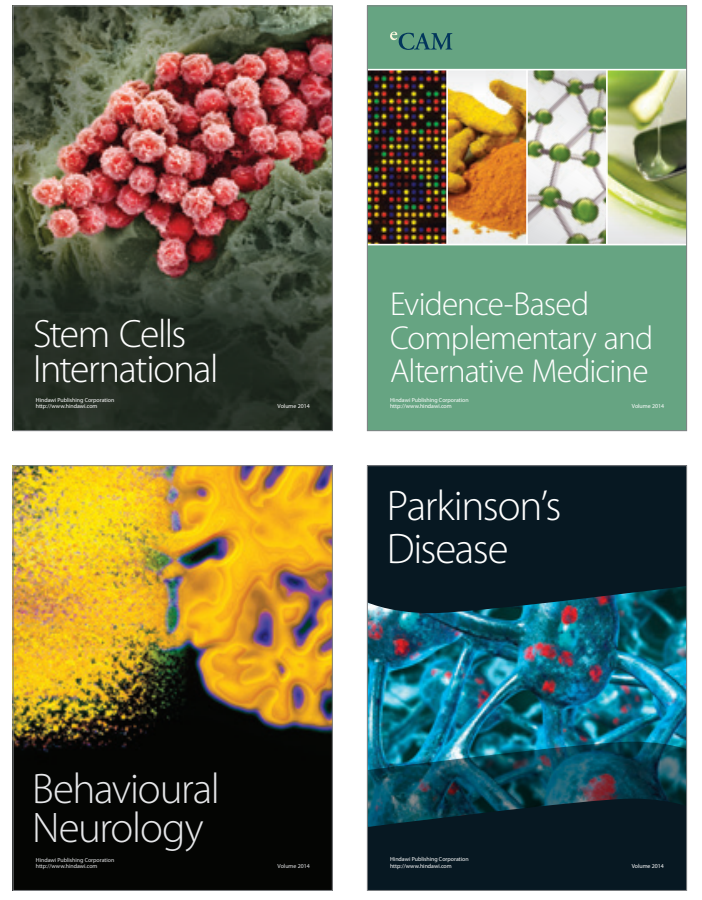
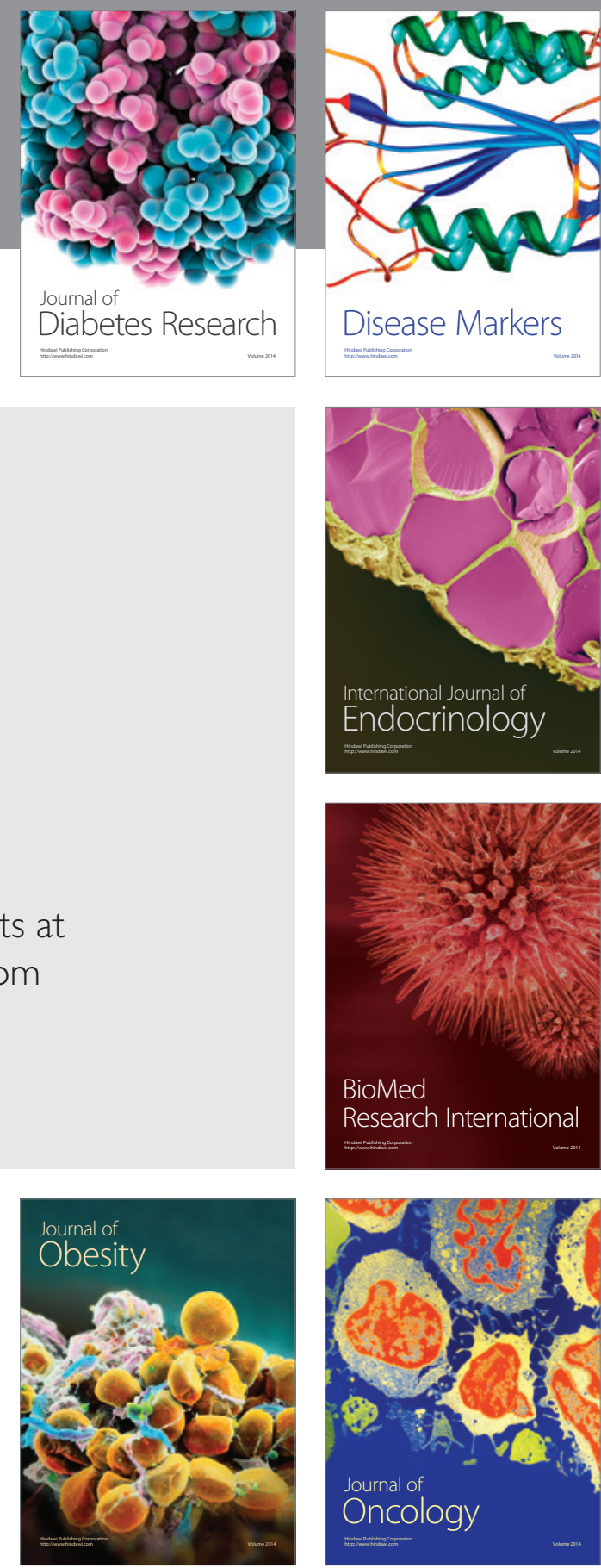

Disease Markers
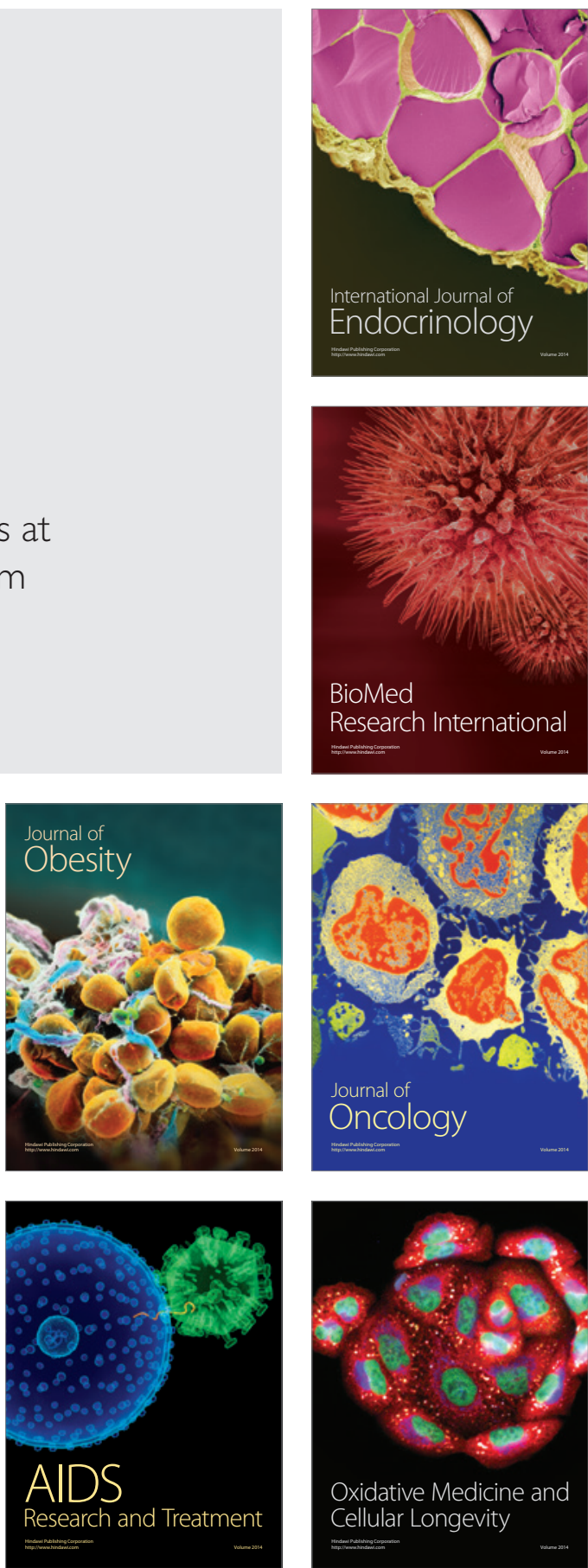